This item was submitted to Loughborough's Research Repository by the author.

Items in Figshare are protected by copyright, with all rights reserved, unless otherwise indicated.

\title{
Thermal performance of additively manufactured polymer lattices
}

\section{PLEASE CITE THE PUBLISHED VERSION}

https://doi.org/10.1016/j.jobe.2021.102243

\section{PUBLISHER}

Elsevier BV

\section{VERSION}

AM (Accepted Manuscript)

\section{PUBLISHER STATEMENT}

This paper was accepted for publication in the journal Journal of Building Engineering and the definitive published version is available at https://doi.org/10.1016/j.jobe.2021.102243

\section{LICENCE}

CC BY-NC-ND 4.0

\section{REPOSITORY RECORD}

Alqahtani, Saad, Hafiz Muhammad Ali, Farukh Farukh, Vadim Silberschmidt, and Karthikeyan Kandan. 2021. "Thermal Performance of Additively Manufactured Polymer Lattices". Loughborough University. https://hdl.handle.net/2134/14061293.v1. 


\title{
Thermal performance of additively manufactured polymer lattices
}

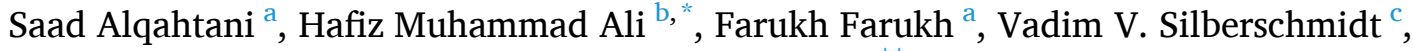 \\ Karthikeyan Kandan ${ }^{\text {a, } * *}$

\footnotetext{
${ }^{a}$ Institute of Engineering Sciences, School of Engineering and Sustainable Development, De Montfort University, The Gateway, Leicester, LE1 9BH, UK

${ }^{\mathrm{b}}$ Mechanical Engineering Department, King Fahd University of Petroleum and Minerals, Dhahran, 31261, Saudi Arabia

${ }^{\mathrm{c}}$ School of Mechanical, Electrical and Manufacturing Engineering, Loughborough University, Loughborough, UK
}

\begin{abstract}
A B S T R A C T
The energy performance of buildings is a key point to achieve the sustainability goals of the modern world. The reduction of the heat loss by incorporating porosity in a monolithic material was studied. To this aim, lattice structures with varying lattice topology and specimen size were synthesised using polymer based additive manufacturing. Commercially available 3D printers and polymer filaments were utilised to manufacture such polymer lattices. Their thermal performance was characterised using a bespoke compact temperature-change hot chamber. A scaling law, based on the experimental results, has been proposed for the first time to predict the Uvalue of polymer lattices by correlating their effective thermal conductivities. It was observed that the lattice's relative density and the sizes of a unit cell and specimen affected significantly the U-value. Also, it was found that polymer-lattice structures can be designed to only allow a conductive mode of heat transfer when their hydraulic diameter was less than $8 \mathrm{~mm}$. The effect of an AM process parameters such as the layer thickness and type of 3D printer on the U-value of the polymer lattices was also characterised and found that they had a mild effect on the U-value of the lattices. Thus, a highly optimised lattice structure, aiming at achieving the higher thermal resistance to make it suitable for energy saving applications, can be obtained using the proposed scaling law.
\end{abstract}

Keywords:

Additive manufacturing

Polymer lattices

Fused filament fabrication

Effective thermal conductivity

U-value

\section{Introduction}

Porous materials have found a significant scientific and technological interest in recent research related to energy-saving concerns in industrial processes and buildings. Such materials consist of a solid matrix with interconnected or closed pores. Thermal conductivity in combination with the specific heat capacity of a solid material defines heat transfer in materials in steady-state and transient situations. As the thermal conductivity value of the air is lower than that of solids, incorporating pores into a solid decreases its thermal conductivity. Thermal performance of a porous material depends on the conductivity of the air within these pores as well as other parameters, including volume fraction of pores and their pattern [1-3]. Cellular materials are a type of porous material with a repeating cell structure and they fall within two categories: foams and lattices. In the former, a gaseous phase is randomly dispersed in a continuous solid medium, whereas the latter consists of a periodic architected cell [4].

As buildings account for about $40 \%$ of the global energy consump- tion and there is a great demand to improve their energy efficiency, this paper is focussed on effectiveness of cellular materials as building blocks [5]. There is an increasing demand to find alternative low-energy, sustainable materials not only to minimise the embodied and operational energy cost of the building but also to reduce the associated carbon footprint [6]. The embodied energy cost arises due to the manufacturing of building products and processes used in initial construction, life cycle management and final demolition of a building, whereas the operational energy cost are associated with the air-conditioning, heating, lighting and operating building equipment [7]. A substantial change in the design of a building materials is required to significantly reduce both embodied and operational energy costs of the building [8]. This paper focuses on such design to develop thermally efficient alternative building blocks for the built environment.

Nature frequently uses cellular materials, thanks to their loadcarrying and weight-optimised structure, for structural and functional demand, e.g. bones. For a long time, the development of artificial cellular materials was aimed at utilizing these outstanding structural

\footnotetext{
** Corresponding author.

* Corresponding author.

E-mail addresses: hafiz.ali@kfupm.edu.sa (H.M. Ali), karthikeyan.kandan@dmu.ac.uk (K. Kandan).
} 
features for various applications. However, the ability of 3D printing to create very intricate forms with precise amount of material makes it perfect for manufacturing lattice materials [9]. So, several studies focused on the mechanical properties and thermal conductivity of 3D printed structures in the literature [10-13]. Some studies aimed at heat transfer performance of lattice-based structures are also found in the literature [14,15]. Nevertheless, most of these studies were related either to hollow bricks (i.e. a very special type of porous materials used for buildings) or heat exchangers. Besides, most of these studies were focussed on market-oriented aspects of these products. Thus, there is a need to study the thermal behaviour of polymer lattices manufactured using 3D printing.

Generally, considerable research was conducted on the thermal performance of cellular and lattice materials. However, most research was focused on metal foams, with isotropic properties and homogeneous lattice structure [16-18], since they can bear moderate load in multiple directions and are commercially available. This research goes further to include anisotropic properties of the foam in analysis of structural and thermal loads $[19,20]$. In addition to that, prior research was more focussed on thermal performance of the lattices during forced-convection fluid flow through them. The aim of these studies was to use the lattices and porous metals as efficient compact heat exchangers. This goal of enhancing thermal conductivity of porous materials for heat exchanger and energy storage applications using forced convention and fillers such as Phase Change Materials was opposite to the study of heat insulation systems which is the focus of this study.

In terms of modelling, there are analytical models which successfully predict the effective thermal conductivity (ETC) of foams and metal lattices [21-24]. However, their validity for polymer lattices manufactured via additive manufacturing is yet to be tested. To the best of authors' knowledge, no work was reported on the mechanism and mode of heat loss as well as on thermal performance of highly ordered 3D printed polymer lattices with enclosed air cavities. The aim of this study was not only to understand the heat transfer mechanisms in polymer based lattice structure with enclosed air cavities but to propose a scaling law to predict the U-value of lattice structures. Thus, this research can provide a comprehensive design resource for engineers seeking to design lattice structures for high thermal performance applications such as in buildings as well as a roadmap for further commercially relevant research activities.

\section{Experimentation}

In order to perform the thermal performance study of lattices, three different types of unit cells i.e. triangular, diamond and hexagonal cells were chosen. One of the major reasons to choose these cell types is their better mechanical performance making them suitable for building applications. Lattice based specimens were developed using additive manufacturing technique due to its simplicity and ability to handle complex shapes. The lattice specimens size as well as the size of their unit cell were defined by the 3D printers' (Prusa i3 MK3s) specifications employed for this study. The wall thickness of the unit cell was kept constant for all the lattice specimens at $0.4 \mathrm{~mm}$, defined the nozzle diameter of the printer. However, the strut length was varied to achieve various cell sizes. The thermal performance of the developed lattice specimens was studied using a technique called temperature-change hot chamber. All thermal experiments were conducted according to ISO 9869 standard.

\subsection{Additive manufacturing of polymer lattices}

A circular specimen, as sketched in Fig. 1a, with a diameter of 120 $\mathrm{mm}$ is periodically filled with lattice unit cells in the $X Y$-plane with a depth $H$ through $Z$-axis. Thus study is focused on triangular, diamond

(a)

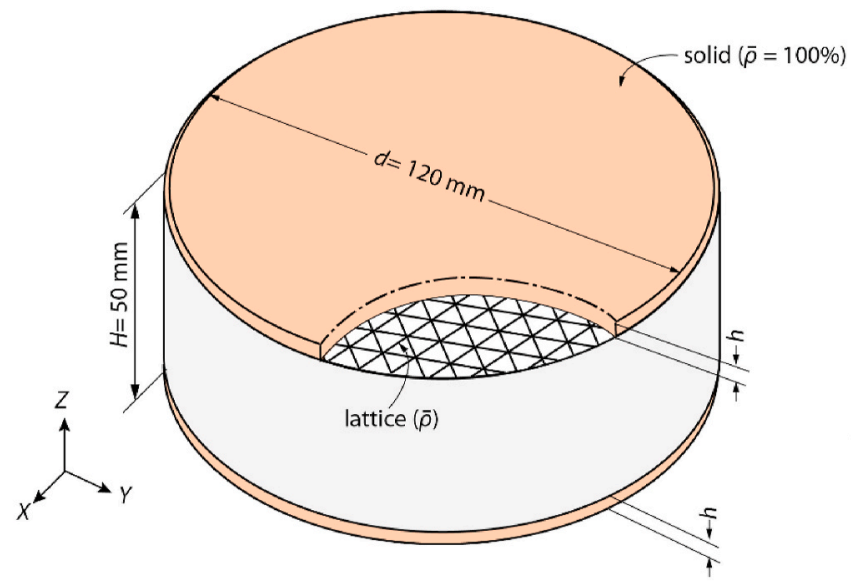

-Not to scale-
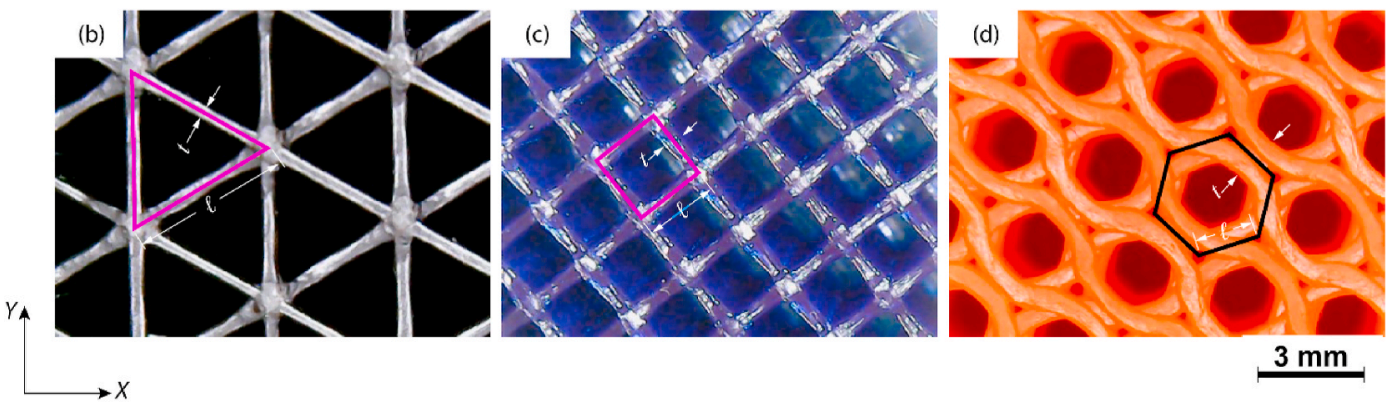

$3 \mathrm{~mm}$

Fig. 1. (a) Sketch showing geometrical details of lattice-based test specimen used in the study. The cutaway in the top of the specimen is introduced to show the lattice topology inside of the specimen. Optical microscopy images of 3D printed triangular (b), diamond (c) and honeycomb (d) lattices. Insets present strut lengths $\ell$ and thicknesses $t$ of each lattice unit cell. 
and hexagonal lattices. In all lattices, the unit cell is relative density $\bar{\rho}$ is characterised by the thickness of struts $(t)$ and their length $(\ell)$ using the following relation:

$\bar{\rho}=A \frac{t}{\ell}$.

The coefficient $A$ depends on the lattice topology and takes values of $2 \sqrt{3}$ for triangular and $2 / \sqrt{3}$ for hexagonal lattices, whereas being 2 for a diamond unit cell. To fabricate the specimen using additive manufacturing, a 3D Computer-Aided Design (CAD) model of the specimen was created in Creo Parametric (version 6.0.2.0), and then imported to Prusa 3D slicing software called Slic3r (version 2.2.0). The wall thickness of lattice struts was kept the same at $0.4 \mathrm{~mm}$, defined by the nozzle diameter of the 3D printer (Prusa i3 MK3s) for each lattice and their strut length $(\ell)$ was varied to fill the material space with a chosen relative density. The optical-microscopy images for the 3D printed lattices with triangular, diamond and honeycomb unit cell as shown in Fig. 1b-d.

Lattices with different relative densities - 20, 40, 60, 80 and 100\% were manufactured with polylactic acid (PLA) 3D printing filaments. The filaments, supplied by Prusa Research, with a nominal diameter of $1.75 \mathrm{~mm}$ were stored in a sealed Pelican container during 3D printing. Additive manufacturing of lattices was performed with a layer thickness of $0.2 \mathrm{~mm}$ for all the lattices. The nozzle and bed temperatures for printing the specimens using PLA were $215^{\circ} \mathrm{C}$ and $60^{\circ} \mathrm{C}$, respectively, as shown in Table 1 . The top and bottom layers of all the specimens were produced with $100 \%$ infill density to yield thickness of $1 \mathrm{~mm}$ (i.e. $h=$ $1 \mathrm{~mm}$ as shown in Fig. 1a) to achieve an enclosed air cavities within the lattice.

\subsection{Thermal testing protocol}

A hot-box calorimeter was used to measure the heat loss through a material by imposing a controlled thermal gradient across the sample employing environmental chambers. This parameter is commonly expressed in terms of $\mathrm{U}$-value of the sample for a given thickness. The Uvalue is the thermal transmittance co-efficient (unit is $\mathrm{W} / \mathrm{m}^{2} \mathrm{~K}$ ) and is the measure of heat loss from hot to cold side of a specimen, assuming the area of $1 \mathrm{~m}^{2}$ and the temperature difference of $1 \mathrm{~K}$. Since polymers are known for lower thermal conductivity compared to metals, the typical test duration for attaining a steady-state heat transfer through a polymer sample showed be significantly longer than that of a metal or a glass in a large environmental chamber. Besides, there is yet no established a method for measuring heat transfer through polymer lattices with enclosed air cavities. To circumvent this problem, a compact temperature-change hot chamber was designed to measure the heat loss. In this testing apparatus, the enclosed area (shown in Fig. 2a) is divided into two warm and cold chambers. These chambers were constructed using graphite expanded polystyrene (EPS) slabs with a thickness of $100 \mathrm{~mm}$ to maintain the adiabatic conditions during the experiment. The external perimeter of the box was insulated with $6 \mathrm{~mm}$ plywood, while the internal walls were insulated with a thermal liner with $10 \mathrm{~mm}$ thick closed-cell foam. The warm and cold chambers were separated by a wellinsulated mask wall made from polystyrene foam slab with a crosssection of $600 \mathrm{~mm} \times 1000 \mathrm{~mm}$ and thickness of $100 \mathrm{~mm}$, see Fig. 2b. A baffle plate was used in both cold and hot chambers to maintain the

Table 1

Nozzle and bed temperature used in Prusa i3 MK3 3D printer for manufacturing lattices.

\begin{tabular}{lll}
\hline Material & Nozzle temperature, ${ }^{\circ} \mathrm{C}$ & Bed temperature, ${ }^{\circ} \mathrm{C}$ \\
\hline Nylon & 240 & 80 \\
PLA & 215 & 60 \\
ABS & 255 & 100 \\
PETG & 240 & 90 \\
\hline
\end{tabular}

uniform temperature distribution in them. A circulating bath (Thermo Scientific AC150-A25 7-12L) filled with ethylene glycol-based coolant was employed to maintain the temperature at a level below ambient in the cold chamber. A silicone heater mat was mounted on the baffle plate and controlled via a regulated DC power supply to increase the temperature in the warm chamber. A circulating fan was also used to maintain a uniform temperature in the warm chamber.

To confirm the validity of the test setup and measurement according to ISO 9869 standard, a calibration test was performed with a polystyrene mask wall with thickness $100 \mathrm{~mm}$ and a known U-value. For this test, the temperatures of $30^{\circ} \mathrm{C}$ and $5^{\circ} \mathrm{C}$ was maintained in a warm and cold chamber, respectively. The steady-state temperature distribution on the surface of the mask wall and air temperature were measured using the thermocouples and resistance temperature detectors (RTDs) in both chambers. These sensors were supplied and factory-calibrated by Thermosense UK with an accuracy of $\pm 0.15{ }^{\circ} \mathrm{C}$ at $0{ }^{\circ} \mathrm{C}$ and $\pm 0.35{ }^{\circ} \mathrm{C}$ at $100{ }^{\circ} \mathrm{C}$ ). During the experiment, thermocouples and RTDs were uniformly distributed on both side-mask walls as shown in Fig. 2b. The measurements were recorded for every $1 \mathrm{~s}$ with gSKIN ${ }^{\circledR}$ DLOG-4231 and National Instruments data logger for $72 \mathrm{~h}$ to comply with ISO 9869 standard. The heat loss through the centre of the specimen was measured using the heat-flux sensor ( $\mathrm{gSKIN}{ }^{\circledR}$-XO 67 7C) with a sensitivity of $7 \mu \mathrm{V} /\left(\mathrm{W} / \mathrm{m}^{2}\right)$. This enabled us to calculate the centre pan Uvalue of the mask wall by using a well-known thermal resistance concept:

$U=\frac{q}{\Delta T}$,

where $q$ is the heat flux per unit area and $\Delta T$ is the measured temperature difference across the specimen. $\Delta T$ was calculated from the two thermocouples probes measuring the specimen temperature on either side of the warm and cold chambers; the probes were located $50 \mathrm{~mm}$ away from the mask wall. From a typical measurement for the mask wall (Fig. 3a); it can be seen that the temperature difference $\Delta T$ attained a steady state approximately $3 \mathrm{~h}$ from the beginning of the experiment and had a mean value of $27.11^{\circ} \mathrm{C}$ during the entire duration of the test. Apparently, the mask-wall facilitated negative heat transfer from warm to the cold chamber throughout the duration of the test with a mean value of heat flux $q=7.2 \mathrm{~W} / \mathrm{m}^{2}$ (Fig. 3a). This implied that the cold and warm chambers were well enclosed and insulated, with almost no heat loss to the environment during the entire duration of the test. Using Eq. (2), the centre pan U-value of the mask wall calculated with the measured temperature difference $\Delta T$ and heat flux, $q$. The time history of calculated U-value (Fig. 3b) for the mask wall demonstrates that the measured U-value of the mask wall remained constants, with a mean value of $0.29 \mathrm{~W} / \mathrm{m}^{2}$ after attaining the steady state. The measured value was in excellent agreement with the standard value of $0.3 \mathrm{~W} / \mathrm{m}^{2}$ for a $100 \mathrm{~mm}$ thick graphite EPS slab. Further calibration tests using singleand double-glazed units with square K-glass panes with a side length of $200 \mathrm{~mm}$ were also performed employing the developed hot chamber. With the mentioned experimental techniques, the U-values of 4.44 and 2.48 were obtained for single-and double-glazed units, respectively. These U-values were in good agreement with the data published elsewhere [25]. For the sake of brevity, time histories of the measured data for temperatures and heat flux related to those calibration experiments are not included here. All the calibration tests demonstrated that the test set-up and measurement protocol conformed to the ISO 9869 standard for testing the U-Value of the coupons. Subsequently, experiments using calibrated hot box were performed to evaluate the thermal insulation capacity of lattice specimens manufactured using 3D printing.

To facilitate the heat-transfer measurements via the polymer lattices, the mask-wall was altered with a provision to mount a lattice specimen (Fig. 2c). A hot-wire knife was used to create a circular recess with diameter of $120 \mathrm{~mm}$ in the centre of the mask wall as shown in Fig. 2c. A silicone sealant was applied around the periphery of the specimen to 
(a)

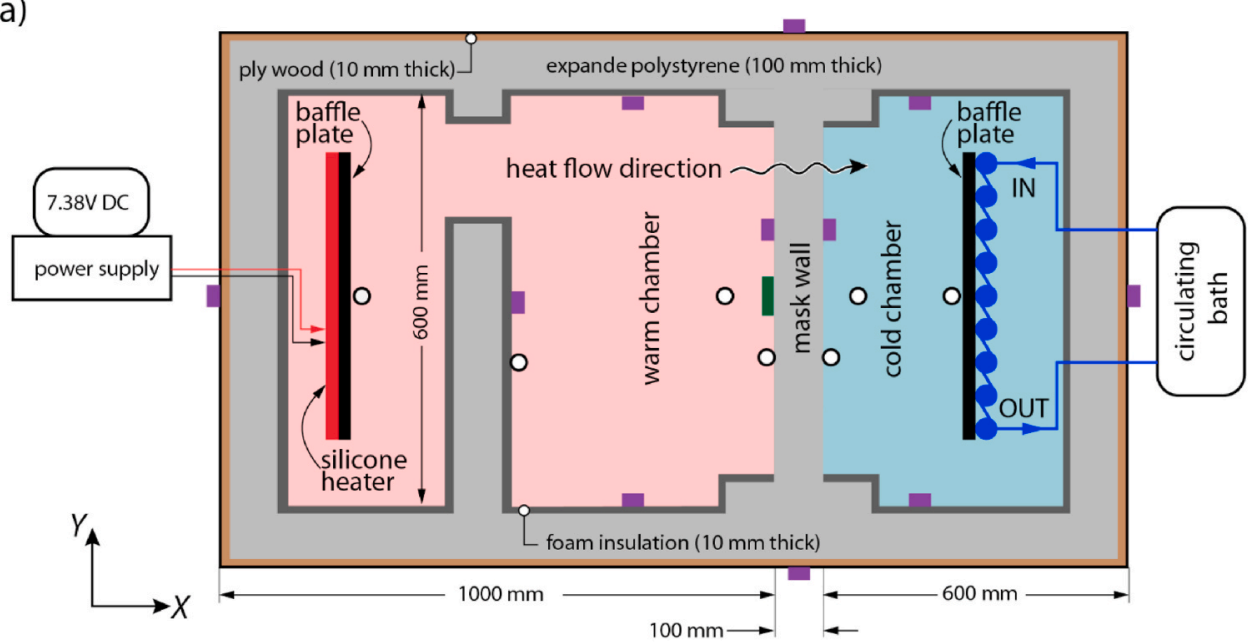

-Not to scale-

(b)

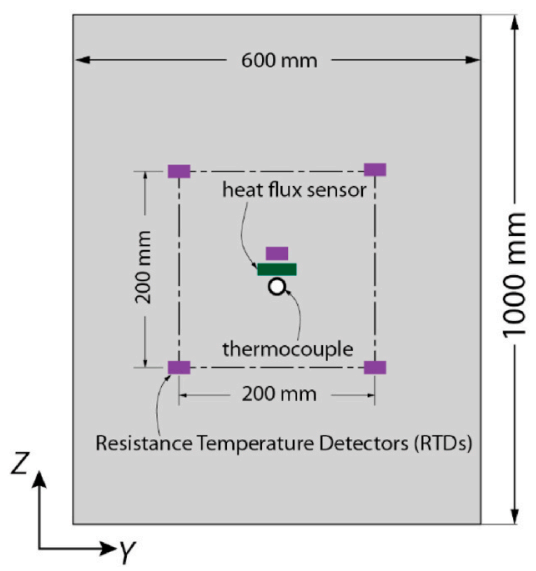

(c)

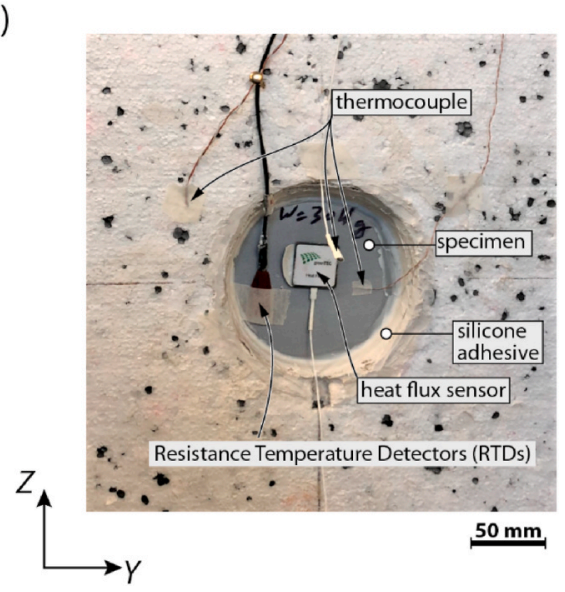

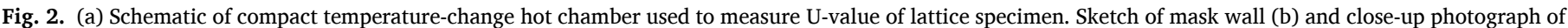

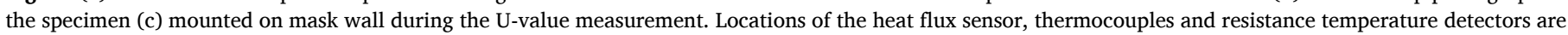
shown in each figure.
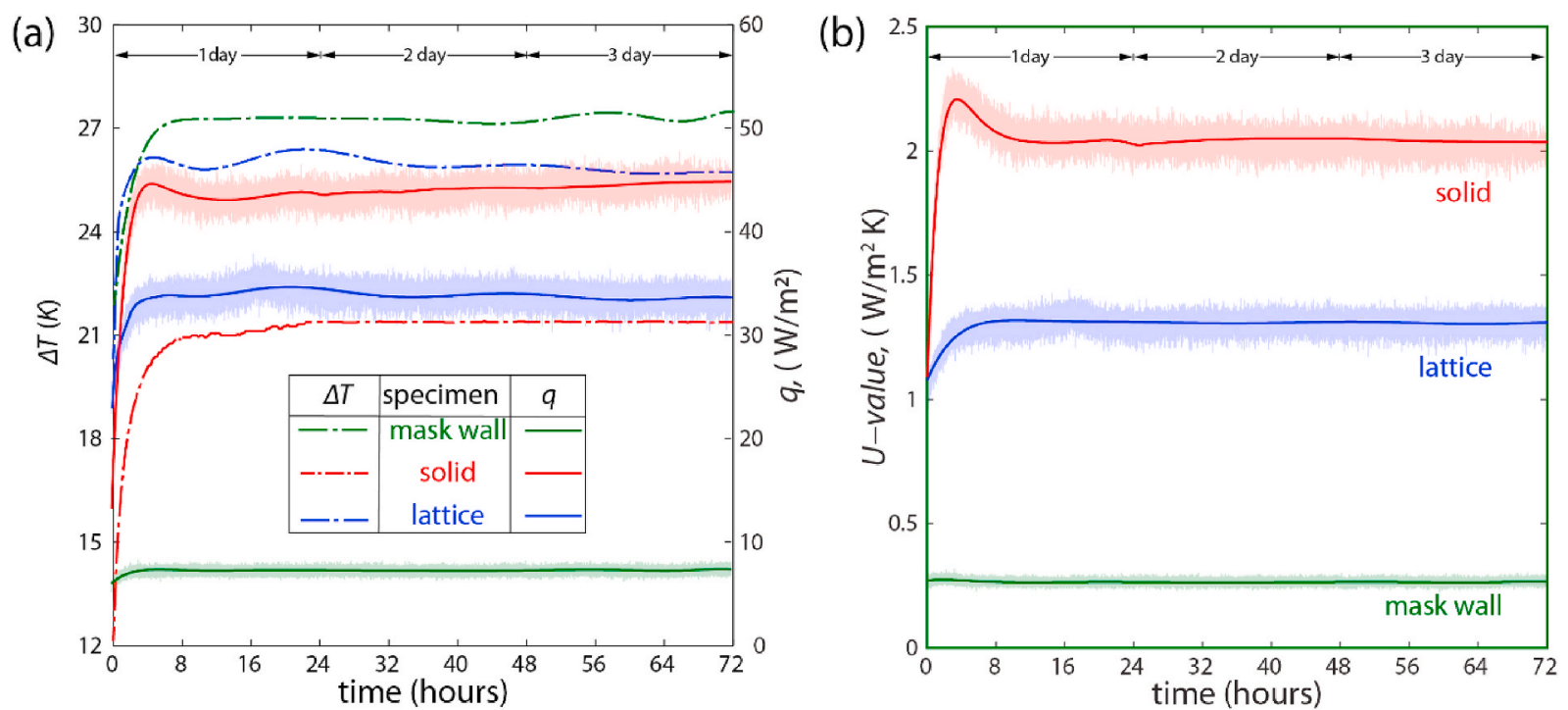

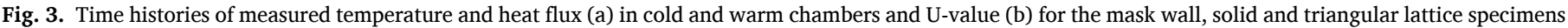
for $\bar{\rho}=40 \%$. The depth of the mask wall was $H=100 \mathrm{~mm}$ while both solid and lattice specimens had the same depth of $H=50 \mathrm{~mm}$. 
adhere it into the circular recess of the mask wall and waited for $24 \mathrm{~h}$ for the curing of the silicone glue before testing. The test procedure outlined above, used for the mask wall, was followed to test the polymer lattice samples.

\section{Results and discussion}

This section outlines the thermal performance of polymer lattices tested in hot-box calorimeter using the method mentioned in Section 3. All the lattice specimens reported in this section were additively manufactured using PLA. In order to keep the discussion tractable, only a triangular lattice is considered initially. The reason to choose triangular lattice lies in that fact that it has superior mechanical performance as compared to other type of lattice structures used for this study.

\subsection{Effect of relative density and unit-cell size}

The measured time histories for temperature difference $\Delta T$, heat-flux $q$ and U-value for triangular-lattice specimens with $\bar{\rho}=40 \%$ and the solid specimen are shown in Fig. 3. Apparently, the steady-state heat transfer was established after approx. $3 \mathrm{hrs}$ from the beginning of the experiment. The mean U-values of $2.17 \mathrm{~W} / \mathrm{m}^{2}$ and $1.30 \mathrm{~W} / \mathrm{m}^{2}$ was measured for solid and lattice specimen, respectively. It is clear that the U-value for the solid specimen was significantly higher than the triangular lattice specimen with $\bar{\rho}=40 \%$, despite both specimens having the same diameter $(d=120 \mathrm{~mm})$ and depth $(H=50 \mathrm{~mm})$. This warranted further investigation of the effect of relative density and depth of the specimens on their thermal performance. For this purpose, the triangular-lattice specimen with varying relative density ranging from $20 \%$ to $100 \%$ ( $100 \%$ corresponds to $3 \mathrm{D}$ printed solid specimen) and varying depth, i.e. $H=8 \mathrm{~mm}, 16 \mathrm{~mm}$ and $50 \mathrm{~mm}$ were subjected to Uvalue test. It was found that the mean $U$-value of triangular lattice specimens increased with increasing relative density and depth (Fig. 4a). With an increase in relative density, the amount of solid material in the lattice grew and, thus, porosity (air pockets) in structure decreased. As the U-value of a solid lattice material is higher as compared to that of air, an increase in the relative density raised the U-value of the lattices. The $\mathrm{U}$-value is a measure of heat loss through a given depth of a particular material. This heat loss occurred via conduction through solid walls of the lattice while via convection and radiation within an enclosed cavity.
It is known that an increase in the depth of the specimen along the heattransfer direction reduces the U-value of the lattices.

The U-value of a material considering three modes of heat-transfer: conduction, convection and radiation can be written as

$U=\frac{1}{\Delta T}\left(q_{\text {conduction }}+q_{\text {convection }}+q_{\text {radiation }}\right)$

As reported in the literature, macro-scale pores in highly porous cellular solids inhibit heat transfer via convective and radiative modes, thus, heat transfer takes place predominantly via conduction through solid walls of the lattices [26,27]. To investigate this hypothesis in the case of polymer lattices, heat transfer by convection and radiation were calculated separately. First, the radiative heat flux through the lattice $q_{\text {radiation }}$ was calculated. It was defined as net flux through the lattice including solid and gas phases.

$q_{\text {radiation }}=A_{s} \sigma\left(T_{h, s}^{4}-T_{c, s}^{4}\right)$,

where $A_{s}$ is the area of the specimen, $\sigma=5.67 \times 10^{-8} \mathrm{~W} / \mathrm{m}^{2} \mathrm{~K}^{4}$ is the Boltzmann's constant, $T_{h, s}$ and $T_{c, s}$ are the specimen's surface temperature measured on the warm and cold sides of the chamber, respectively. Based on Eq. (4), $q_{\text {radiation }}$ was estimated as $1.19 \mathrm{~W} / \mathrm{m}^{2}$ for the triangular lattice specimen with $\bar{\rho}=40 \%, H=50 \mathrm{~mm}$ and $A_{s}=0.0113 \mathrm{~m}^{2} . T_{h, s}=$ $305.20 \mathrm{~K}$ and $T_{c, s}=287.02 \mathrm{~K}$ were maintained across the specimen during the test. Thus, the contribution by radiation heat transfer ( $q_{\text {radiation }}$ ) to the U-value was calculated as $0.0667 \mathrm{~W} / \mathrm{m}^{2} \mathrm{~K}$. The measured total U-value of this specimen using the hot-box calorimeter was $1.2614 \mathrm{~W} / \mathrm{m}^{2} \mathrm{~K}$. Thus, the contribution of radiation heat-transfer as compared to the total heat transfer from this particular lattice specimen was rather low. Other thickness of lattices and relative densities (reported in Fig. 4) were also tested under conditions similar to those used for the triangular-lattice specimens (i.e. $\bar{\rho}=40 \%, H=50 \mathrm{~mm}$ and $A_{s}=$ $\left.0.0113 \mathrm{~m}^{2}\right)$. Hence, apparently the radiative mode of heat transfer was not a major contribution to the heat loss in the 3D printed polymer lattices tested in this study.

The convection heat transfer through the air inside the structure depends on various factors including the temperature gradient across the specimen and the size of the air pocket. For the stagnant air inside the structure the effect of convection vanishes. Still the air moves when the temperature difference across specimen is large enough or air-pocket
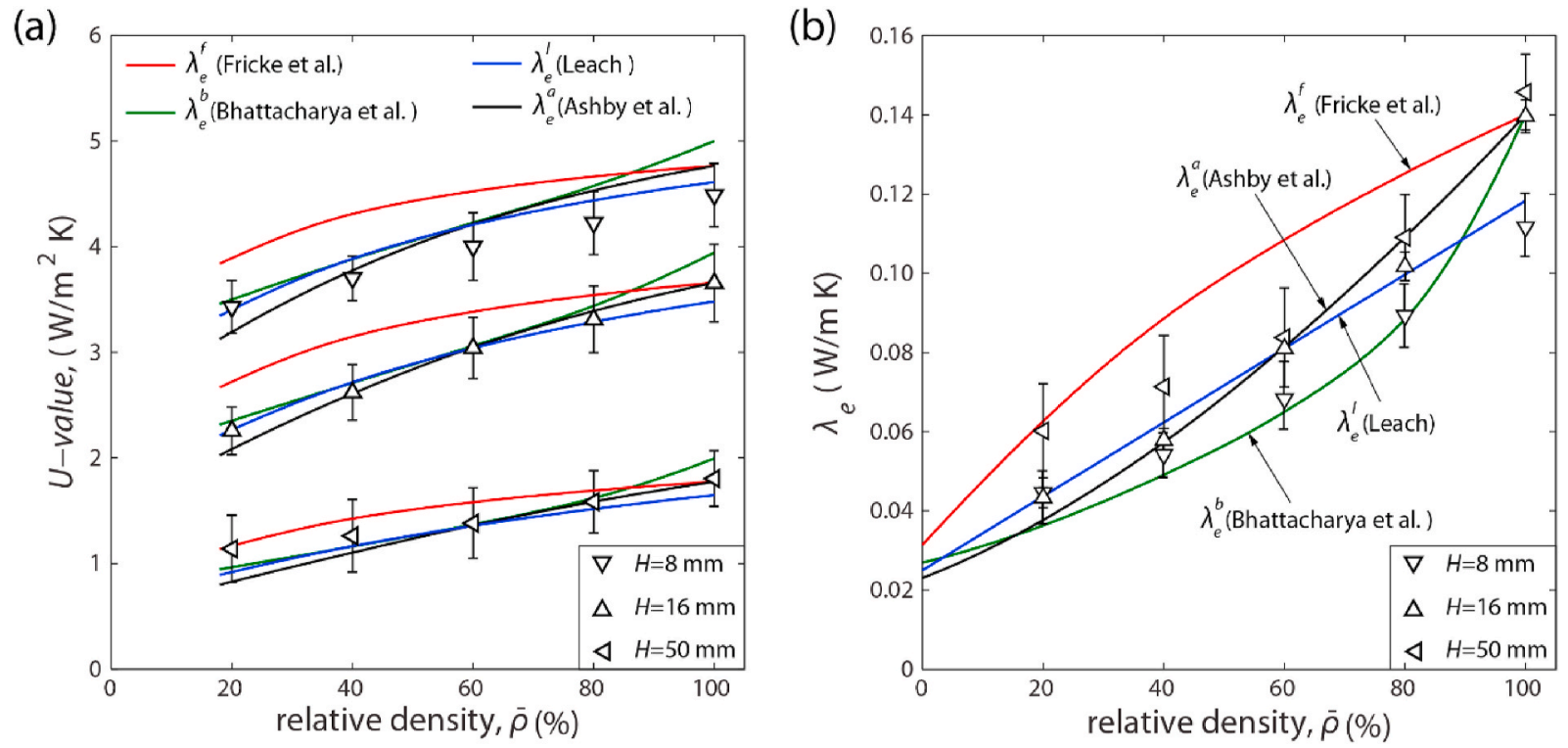

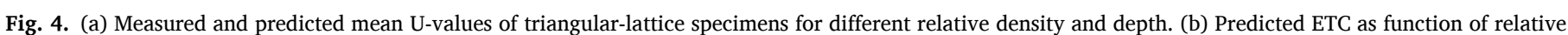

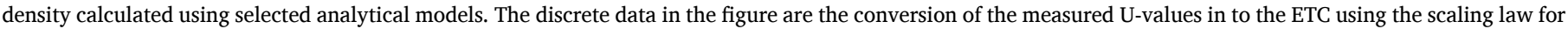
triangular lattices. The $100 \%$ relative density corresponds to the $3 \mathrm{D}$ printed solid specimen. 
size is big. The Grashof number, Gr, which can be employed to investigate the significance of convection within or between the enclosed pores of the periodic lattices, is used to predict the convection-related heat transfer in this study [26]. It is defined as the ratio of buoyancy to viscous forces and can be expressed for the enclosed pore with a hydraulic diameter, $D_{h}$;

$G r \approx \frac{g \nabla T D_{h}^{3} \rho^{2}}{T_{\text {avg }} \eta}$

where $g$ is the acceleration due to gravity, $\rho$ and $\eta$ are the density and dynamic viscosity of the air, respectively, $T_{\text {avg }}$ is the average air temperature the pore and $\nabla T$ is the measured temperature difference across the specimen. The hydraulic diameter $D_{h}$ for the 3D printed lattices can be expressed as

$D_{h}=\frac{4 \ell \sqrt{1-\bar{\rho}}}{c_{a}}$

where $\bar{\rho}$ is the relative density of the lattice, $\ell$ is the strut length and $c_{a}$ is the shape factor, which depends on the lattice topology: $c_{a}$ is $6.93,4$ for triangular, diamond lattices while 2.31 for honeycomb lattice, respectively [28]. Eq. (6) was used to estimate the pore diameter $D_{h}=1.634 \times$ $10^{-3} \mathrm{~m}$ for triangular lattice with $\bar{\rho}=40 \%$ tested under the following test condition: $g=9.81 \mathrm{~m} \mathrm{~s}^{-2}, \rho=1.293 \mathrm{~kg} \mathrm{~m}^{-3}, \eta=1.849 \mathrm{~kg} \mathrm{~m}^{-1} \mathrm{~s}^{-1}$, $T_{\text {avg }}=290.6 \mathrm{~K}$ and $\nabla T=19.43 \mathrm{~K}$. For this particular specimen (where $\bar{\rho}=40 \%, H=50 \mathrm{~mm}$ and $A_{s}=0.0113 \mathrm{~m}^{2}$ ), the estimated Grashof number using Eq. (5) equals to 14.32. The natural convection within the pores of the lattice is expected to be significant when this value (called critical Grashof value) reaches 1000 [26]. However, the estimated $G r$ for the specimen with the triangular lattice is an order of magnitude lower than the critical Grashof number. This indicates that the convective heat transfer does not play any significant role in heat transfer through the specimen. Other lattice specimens reported in Fig. 4a (with different thicknesses and relative densities) were also tested at the test conditions similar to those of this lattice specimen. However, the hydraulic diameters of those lattices were different, as the strut length of individual lattices scales with relative density, resulting in different Grashof numbers. Still, for all the lattices used (Fig. 4a) Grashof number values were less than the critical value, meaning heat transfer through convection was negligible.

It raises a question of the optimum hydraulic diameter restricting the convective heat transfer in the 3D printed lattices. Knowing the optimum $D_{h}$ of a lattice structure for the given relative density has a practical implication: from the manufacturing point of view, larger unit cells are better since they enable rapid production of lattices. At the same time, big unit cells may lead to convection heat transfer as discussed. So, for a given relative density, the unit cell size of a lattice structure (i.e. the size of each air pocket inscribed by the lattice's unit cell) can be defined by appropriately choosing the strut length and thickness based on Eq. (1). It is worth mentioning here that the optimum size of the unit cell needs to be scaled up should the lattice specimen size is being scaled up from coupon to larger panel size. It will not only help to maintain the relative density of the lattice but to provide better thermal performance of the panel by avoiding convection heat transfer by the air entrapped by the cell walls.

To obtain the optimum hydraulic diameter of a lattice structure, further experiments were performed on triangular-lattice specimens with a varying size of the unit cell for a fixed specimen depth of $50 \mathrm{~mm}$, $\bar{\rho}=40 \%$ and $A_{s}=0.0113 \mathrm{~m}^{2}$. The boundary conditions were kept constant in all the experiments. To maintain the constant relative density of the specimen, the cell size (i.e. its hydraulic diameter) was varied $\left(D_{h}=1.62-45.54\right)$ by choosing the appropriate thickness, $t$ and length of the strut, $\ell$. The geometrical details of these lattices are reported in Table 2. These experiments were performed in a way that the heat transfer due to conduction and radiation was constant; the only variable was convective heat transfer.

It was found that the U-value of specimens $a-c$ (in Table 2) increased with an increase in $D_{h}$ (Fig. 5a) whereas the specimens $d$-f had a constant U-value of $1.26 \mathrm{~W} / \mathrm{m}^{2} \mathrm{~K}$ remaining independent of $D_{h}$. The Grashof number was also calculated; its value increased with the rise of $D_{h}$. As the Grashof number exceeds 1000 for a lattice structure, convective heat transfer can take place [26]. The black line delineating this critical Grashof number is highlighted in Fig. 5a. For the specimens $a-c$, $G r>1000$, indicating that the convective heat loss in the lattice pores prevailed over conductive heat transfer in the solid walls of the lattice. However, $G r<1000$ for specimens $d-f$ indicates that the conduction via lattice walls dominated the heat transfer, whereas the convective mode offered a negligible contribution. Since the effective thermal conductivity of these lattices was same without contribution from convective heat transfer, the U-value of these lattices was independent of the hydraulic diameter. It is worth noticing that the specimen $d$ had the critical hydraulic diameter of $7.14 \mathrm{~mm}$ which delineate the two regimes of heat transfer. The highest hydraulic diameter of all the triangular lattice specimens reported in Fig. 4a is $3.24 \mathrm{~mm}$, sufficiently lower than the critical hydraulic diameter. Therefore, as concluded earlier, the primary heat transfer mechanism for these polymer lattices was governed by the conductive mode.

Importantly, for this type of lattice structure, with conduction being a major mode of heat transfer, effective thermal conductivity (ETC) can be predicted by employing well-known theoretical models developed for ordered periodic lattices. This calculation is given in next section. For the sake of completeness, a brief description of those theoretical models is discussed below.

\subsection{Effective thermal conductivity and scaling law}

The ETC of the lattice $\lambda_{e}^{b}$ can be computed using a simple rule of mixture considering both a solid matrix and an air cavity which is aligned parallel and perpendicular to the heat flow direction within the lattice is as follows;

$\lambda_{e}^{b}=\left[\bar{\rho} \lambda_{s}+(1-\bar{\rho}) \lambda_{a}\right]+\left[\frac{1-C}{\frac{\bar{\rho}}{\lambda_{s}}+\frac{(1-\bar{\rho})}{\lambda_{a}}}\right]$,

Table 2

Type of specimen and its geometrical details (relative density 40\%).

\begin{tabular}{|c|c|c|c|c|c|c|}
\hline Lattice type & Specimen name & Number of cells, $\boldsymbol{n}$ & Strut length, $\ell(\boldsymbol{m m})$ & Strut thickness, $\boldsymbol{t}(\boldsymbol{m m})$ & $\Delta \boldsymbol{T}_{s},{ }^{\circ} \mathrm{C}$ & $\begin{array}{l}q, \\
W / m^{2}\end{array}$ \\
\hline \multirow[t]{5}{*}{ triangle } & a & 1 & 104 & 8.0 & 12.89 & 42.1 \\
\hline & b & 13 & 50 & 5.77 & 14.27 & 33 \\
\hline & d & 72 & 16 & 1.88 & 16.10 & 24 \\
\hline & $\mathrm{f}$ & 335 & 8.66 & 1.0 & 14.83 & 22.1 \\
\hline & $\mathrm{g}$ & 2082 & 3.66 & 0.42 & 17.98 & 28.3 \\
\hline diamond & $\mathrm{f}$ & 2698 & 2.10 & 0.42 & 17.39 & 24.3 \\
\hline
\end{tabular}



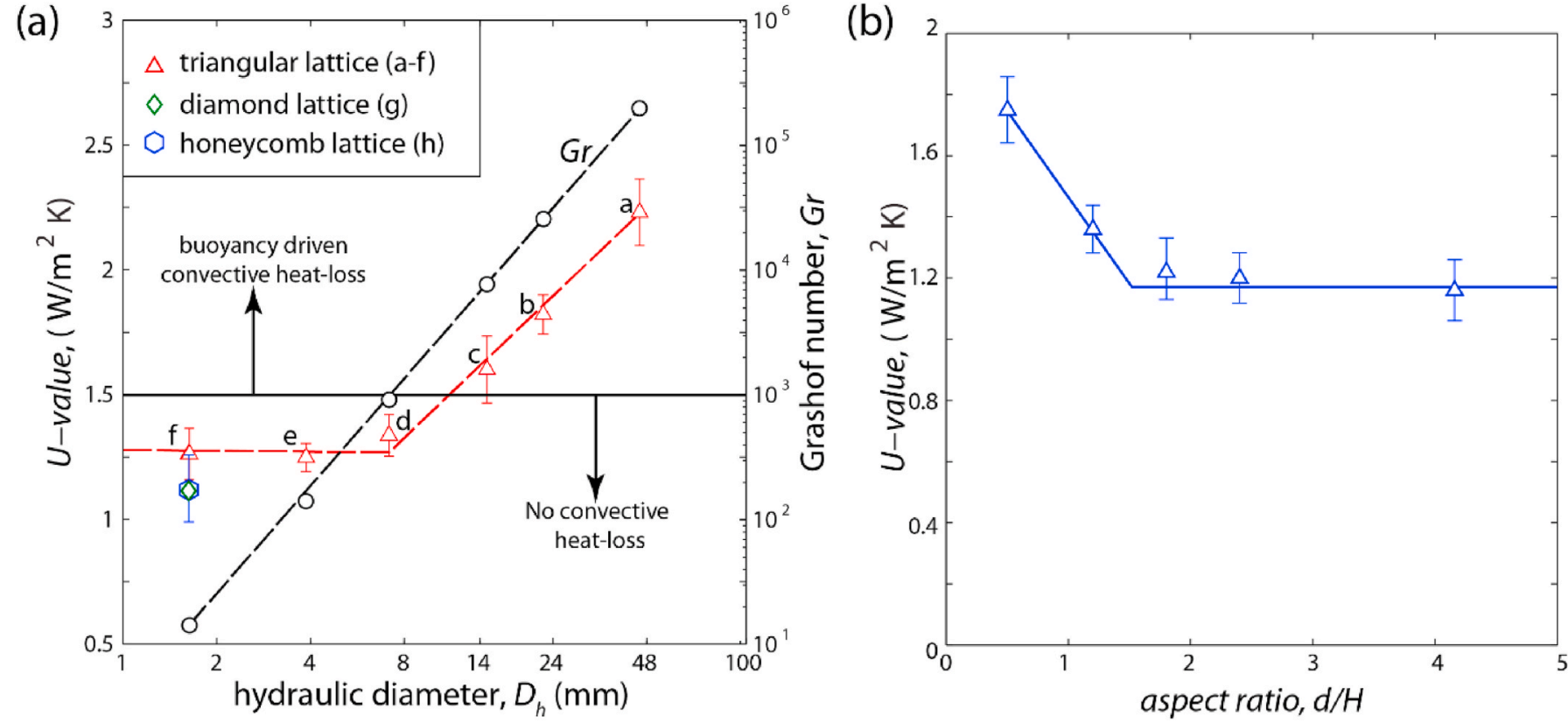

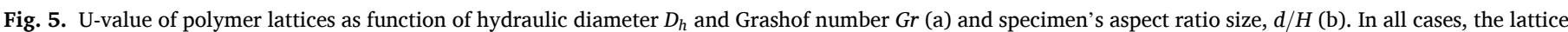
relative density $\bar{\rho}=40 \%$ and depth, $H=50 \mathrm{~mm}$ were fixed.

where $\lambda_{a}$ and $\lambda_{s}$ are the thermal conductivity of the air and a solid constituent of the lattices, respectively. The constant $C=0.35$ is required as a fitting parameter to predict the ETC of the high-porosity aluminium and vitreous carbon foams, filled with air and water [21].

On the other hand, Fricke, et al. is formulation [22] for the $\operatorname{ETC} \lambda_{e}^{f}$ of cellular solids only relies on the speed of sound of both solid and porous media and can be written as

$\lambda_{e}^{f}=\lambda_{s} \bar{\rho}\left(\frac{v_{p}}{v_{s}}\right)$

where, $v_{p}$ and $v_{s}$ is the speed of sound in porous and solid media, respectively.

In closed-cell cellular solids, the lattice walls account for the main part of the heat transfer compared with the trapped air [24], therefore, Ashby [24] proposed the ETC, $\lambda_{e}^{a}$ of cellular solids is as

$\lambda_{e}^{a}=\frac{1}{3}\left[\bar{\rho}+2 \bar{\rho}^{3 / 2}\right] \lambda_{s}+[1-\bar{\rho}] \lambda_{a}$.

While the above models are based on the mechanistic approach i.e., considering the distribution of solid material in the lattice against the heat flow path, there is an alternative self-consistent field theory to account for the unique material behaviour of the heat transfer [29]. Therefore, Leach et al. [23] proposed the ETC, $\lambda_{e}^{l}$ of cellular media using self-consistent field theory can be expressed as

$\lambda_{e}^{l}=\frac{2}{3} \lambda_{s}+\lambda_{a}$.

The ETCs of 3D printed polymer lattices were computed employing the above theoretical models by choosing $\lambda_{a}=0.025 \mathrm{~W} / \mathrm{mK}$ and $\lambda_{s}=$ $0.14 \mathrm{~W} / \mathrm{mK}$ for air and PLA 3D printing filament, respectively. The results for the variation of ETC with relative density $\bar{\rho}$ for the triangular lattice are plotted in Fig. 4b. Evidently, the ETC $\lambda_{e}^{b}$ and $\lambda_{e}^{f}$ (based on the simple rule of mixture and speed of sound in both cellular and solid media) provided lower and upper bounds, respectively. The trends for ETC $\lambda_{e}^{a}$ and $\lambda_{e}^{l}$ (based on the mechanistic approach and self-consistent field theory) were a similar between these bounds. In all the cases, the ETC grew with the increase of relative density of the lattices. Obviously, $\lambda_{e}=\lambda_{s}$ when $\bar{\rho}=100 \%$ corresponding to the $3 \mathrm{D}$ printed solid specimen.
In order to link the effective thermal conductivity of the specimen with its U-value, a scaling law is proposed based on the fact that the heat loss through the polymer lattices is mainly governed by the conductive mode of heat transfer. For a triangular lattice $U$-value $U_{l}$ scaling law is:

$U_{l}=\frac{B}{\left(\frac{1}{U_{s}}+\frac{H}{\lambda_{e}}\right)}$,

where $U_{s}$ is the U-value of the solid specimen for a given depth $H$. The constant $B$ is required to account for the lattice topology; it has a value of $3 / 2$ for the $3 \mathrm{D}$ printed triangular lattice. The results of the predicted $\mathrm{U}$ values of the triangular lattice specimens with different depth as a function of relative density are also plotted in Fig. 4a. The scaling law using ETC $\lambda_{e}^{b}$ and $\lambda_{e}^{f}$ provided the lower and upper bounds, respectively. On the other hand, the measured U-value of triangular lattices were reasonably predicted based on $\lambda_{e}^{a}$ and $\lambda_{e}^{l}$. To gauge the accuracy of the predicted ETC of the lattices, the scaling law reported in Eq. (11) was used to estimate the ETC of lattices employing the measured U-value of lattices and reported in Fig. 4b. It demonstrates that the estimated ETC of lattices was independent of specimen's thickness. Besides, the ETC predictions followed the general trend of the data, i.e. increase in value with relative density. Thus, the proposed scaling law is critical to explore the design space of a lattice specimen for thermal applications. There are several models available in the literature to predict the mechanical design space of the lattices $[24,30]$. To the authors' knowledge, this is the first attempt to predict the U-value of the additively manufactured polymer-based lattices.

\section{Impact factor analysis on thermal performance of lattices}

The U-value of a 3D printed lattice structure is a function of its geometrical features as well as printing-process variables. Three different lattice structures: triangular, diamond and honeycomb were printed for this purpose. The relative density for all the specimens was kept constant at $40 \%$ for direct comparison. Quantitative analysis of the effect of various parameters on the U-value of the 3D printed lattice specimens was performed and given in this section. 


\subsection{Effect of specimen size and lattice topology}

To verify that the measured U-value of the lattices was unaffected by the specimen size, further tests were performed with triangular lattices with fixed $\rho=40 \%$ and selected aspect ratio, $A R=d / H$. Here, $\mathrm{H}$ was fixed at $50 \mathrm{~mm}$ and $d$ ranged from $25 \mathrm{~mm}$ to $208 \mathrm{~mm}$. It was found that the U-value was independent of the specimen size when $A R>1.8$ (Fig. 5b) which correspond to the specimen diameter of $90 \mathrm{~mm}$. The level of $A R$ was maintained at 2.4 (corresponding to the specimen's diameter of $120 \mathrm{~mm}$ ) for all the specimens to asses their U-values in this study; therefore, the specimen size did not influence the U-value.

To investigate the effect of lattice topology on the U-value, further experiments were performed with diamond and honeycomb unit cells. These lattice specimens also had fixed $H=50 \mathrm{~mm}$ and $\bar{\rho}=40 \%$ (their geometry details are listed in Table 2). The measured U-values of these lattices are also plotted in Fig. 5a to compare with those for triangular lattices. As observed, the diamond and honeycomb lattices exhibited approximately $13 \%$ lower U-value compared to that of triangle lattices. These tests revealed that the U-value of the lattices could be also controlled by an appropriate choice of topology of their unit cell.

\subsection{Effect of additive manufacturing process}

It is known that process variables in 3D printing play a vital role in defining not only the mechanical behaviour but also thermal performance of the final product. FDM introduces some internal voids and pores into fabricated thermoplastics giving rise to potential variation in the thermal behaviour of printed parts, especially produced with different printers. To investigate the effect of printing quality of the parts in terms of their accuracy as a function of their thermal behaviour, commonly available desktop 3D printers such as Prusa i3 MK3, 3DGence P255 and Ultimaker and 3DGence Industry F340 printers were used in our tests. While the 3D printing parameters (i.e. layer thickness, fill density, nozzle diameter, heat-bed temperature) were maintained as reported in Table 1 for all 3D printers, the ambient temperature of the print volume for industry F340 printer was equal to the heat-bed temperature. Triangular-lattice specimens with fixed $H=50 \mathrm{~mm}$ and $p=$ $40 \%$ were fabricated for direct comparison. It was found that the measured U-values were affected by the quality of 3D printed parts produced with various types of 3D printers (Fig. 6); however, the effect was falling mostly within a statistical scatter range. Therefore, it can be concluded that different 3D printers had only an insignificant influence

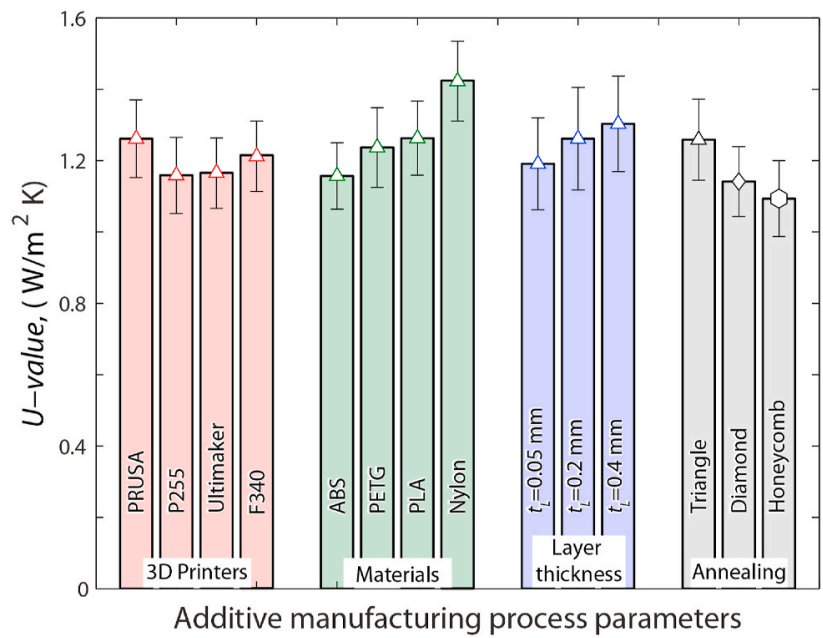

Fig. 6. Effect of AM parameters on U-value of lattices manufactured with commercial 3D printers, various digital materials, layer thickness and lattice topology. Lattice relative density and depth were fixed at $40 \%$ and $50 \mathrm{~mm}$, respectively. on the U-value of triangular lattices.

\subsection{Influence of lattice material}

To investigate the effect of materials on the thermal-transmittance response of 3D printed polymer lattices, most commonly available filaments such as acrylonitrile butadiene styrene (ABS), polylactic acid (PLA), polyethylene (PETG) and polypropylene (PP) were used to fabricate the triangular lattices. Prusa Research supplied the PLA, ABS, PETG and PETG 3D printing filaments while Mark Forged supplied the PP filament. The highest U-value of $1.4239 \mathrm{~W} / \mathrm{m}^{2} \mathrm{~K}$ was observed for the lattice made of PP filament (Fig. 6), while the ABS lattice had a $23 \%$ lower U-value. The other lattices made from PLA and PETG had similar $\mathrm{U}$-values of $1.42 \mathrm{~W} / \mathrm{m}^{2} \mathrm{~K}$. These results were consistent with the reported thermal conductivity of the ABS, PLA and PETG filaments that varied in the range of $0.10-0.20 \mathrm{~W} / \mathrm{m} \mathrm{K}$, whereas the PP filament had a higher thermal conductivity of $0.25 \mathrm{~W} / \mathrm{m} \mathrm{K}$ [31].

\subsection{Influence of layer thickness and post-processing}

It is well-known that the layer thickness plays a crucial role in AM to governing the properties of $3 \mathrm{D}$ printed parts. To investigate its effect on the U-value of the PLA lattices, triangular-lattice specimens were fabricated with different layer thicknesses: $50 \mu \mathrm{m}, 200 \mu \mathrm{m}$ and $400 \mu \mathrm{m}$. For these parameters, the nozzle of the Prusa MK3 printer was changed respectively. In all specimens, the $40 \%$ of relative density and depth of $50 \mathrm{~mm}$ was maintained for direct comparison. The measured U-value was $8.5 \%$ higher for layer thickness of $50 \mu \mathrm{m}$ (Fig. 6) compared to that for $400 \mu \mathrm{m}$. This could be a result of air voids in solid walls. The lower layerthickness settings in the $3 \mathrm{D}$ printing process have a higher proportion of the air voids. To study this effect a bulk PLA specimen with diameter $120 \mathrm{~mm}$ and depth of $50 \mathrm{~mm}$ was prepared by moulding the PLA filaments in a furnace. Its measured U-value was $2.06 \mathrm{~W} / \mathrm{m}^{2} \mathrm{~K}$, whereas the 3D printed solid specimen (100\% relative density) exhibited $1.81 \mathrm{~W} /$ $\mathrm{m}^{2} \mathrm{~K}$ during the test. This confirmed that the air voids in 3D printed solid walls and layered structure of printed specimen provided additional thermal resistance. It is worth noticing that the amount of reduction in $\mathrm{U}$-value of the printed specimen depends upon the number of voids and gaps in the specimen. The more voids in the specimen, the lower will be its thermal conductivity.

Additive manufacturing is the thermal process, which introduces residual stresses in a sample. Therefore, the 3D printed samples should be subjected to post-manufacture heat-treatment process such as annealing to relive thermal residual stress and improve the crystallinity of the material. To assess the effect of annealing process on the U-value of the 3D printed PLA lattices, lattices with three different topologies of their unit cells were annealed following the protocol proposed in Ref. [32]. The samples were placed inside a non-vacuum oven and heated at a rate of $5^{\circ} \mathrm{C} / \mathrm{min}$. After soaking the samples at $100^{\circ} \mathrm{C}$ for 10 min, they were air-cooled to room temperature. The U-value ranking of the lattices remained the same for both annealed and non-annealed specimens (Fig. 6): triangular lattices had higher U-value than the lattices with diamond and honeycomb topologies.

\subsection{Uncertainty analysis}

The error bars in all the figures with experimental results denoting the variation of the measurement were obtained from at least three samples tested over a time period of $72 \mathrm{~h}$. The uncertainties in the $\mathrm{U}$ value of the $3 \mathrm{D}$ printed polymer lattices were governed by the following factors: the additive manufacturing of lattices, compact temperaturechange hot chamber and effective thermal conductivity.

The lattice strut length $\ell$, wall thickness $t$ and mass $m_{s}$ of the AM lattice determine the relative density of a lattices and it can be expressed as function of these: 
$\bar{\rho}=f\left(\ell, t, m_{s}\right)$

The uncertainty of the relative density, $\bar{\rho}$ can be estimated as

$\frac{\delta \bar{\rho}}{\bar{\rho}}=\sqrt{\left(\frac{\delta \ell}{\ell}\right)^{2}+\left(\frac{\delta t}{t}\right)^{2}+\left(\frac{\delta m_{s}}{m_{s}}\right)^{2}}$.

The uncertainty function for the compact temperature-change hot chamber employed to measure the U-value of the 3D printed lattices depends on the errors in measuring the heat flux, $q$, surface temperature of the specimen at warm $\left(T_{h, s}\right)$ and cold $\left(T_{c, s}\right)$ sides, as well as the difference in ambient air temperature across the specimens, $\nabla T$. It also includes the diameter of the specimen. Thus, the uncertainty in the Uvalue measurement can be expressed as

$U_{l}=f\left(q, T_{h, s}, T_{c, s}, \nabla T, \mathrm{~d}\right)$

The uncertainty of the U-value can be estimated as:

$\frac{\delta U_{l}}{U_{l}}=\sqrt{\left(\frac{\delta q}{q}\right)^{2}+\left(\frac{\delta T_{h, s}}{T_{h, s}}\right)^{2}+\left(\frac{\delta T_{c, s}}{T_{c, s}}\right)^{2}+\left(\frac{\delta \nabla T}{\nabla T}\right)^{2}+\left(\frac{\delta d}{d}\right)^{2}}$

The specimen depth $H$, solid specimen's U-value $U_{s}$ and effective thermal conductivity of the lattice $\lambda_{e}$ define the uncertainty function of the scaling law, is used to predict the U-value of the lattices:

$U_{l}=f\left(U_{s}, \bar{\rho}, H, \lambda_{e}\right)$.

The uncertainty of the U-value can be calculated as

$\frac{\delta U_{l}}{U_{l}}=\sqrt{\left(\frac{\delta U_{s}}{U_{s}}\right)^{2}+\left(\frac{\delta \bar{\rho}}{\bar{\rho}}\right)^{2}}+\left(\frac{\delta H}{H}\right)^{2}+\left(\frac{\delta \lambda_{e}}{\lambda_{e}}\right)^{2}$.

Eqs. (13), (15) and (17) were used to estimate the uncertainty of the relative density, measured and predicted U-value; it was found to be less than $7.4 \%, 6.8 \%$ and $9.3 \%$, respectively. This shows the repeatability of the $\mathrm{U}$-value results obtained in this study.

\section{Conclusions}

Thermally efficient building blocks formed by periodic lattice structure were successfully manufactured with 3D printing. For the first time, this study highlights the effect of parameters on the U-value of the 3D printed polymer lattices. The main conclusions from the study are as follows:

- The spatial distribution of material using the periodic lattice architecture can offer a significant thermal-insulation response compared to that of a monolithic block. The relative density of the lattice and specimen's depth can be used to obtain the desired U-value for polymer lattices.

- For a given relative density of the lattice, the unit-cell size governs the mode of heat transfer through the specimen and thus, determines the U-value of a lattice specimen. While the radiative heat transfer offers a negligible contribution to the overall heat transfer for the test conditions outlined in the paper, the hydraulic diameter of polymer lattices uniquely characterises the convective mode of heat transfer. The critical hydraulic diameter of the lattices should be less than 8 $\mathrm{mm}$ to avoid the convective mode of heat transfer.

- Since the conductive mode of hear transfer dominates the thermal transmittance of the polymer lattices, their effective thermal conductivity can be adequately predicted using phenomenological models based on the mechanistic approach and self-consistent field theory.

- The proposed scaling law for prediction of the U-value of polymer lattices by correlating their effective thermal conductivity and the specimen's depth adequately captured the measured U-value of the polymer lattice as a function of relative density.
- Parameters of the additive manufacturing process such as the layer thickness, layer height, type of 3D printers, filament materials and post-processing temperature did not affect considerably the U-value of the polymer lattice.

\section{Future work}

Our work presented here not only helps to explore the thermal design space of additively manufactured lattice structures but also lays a foundation for utilizing them as an alternative building block for constructing an energy-efficient built environment. Further work is required to investigate the thermal transmittance of lattices with complex structure and made from a range of other materials. Manufacturing of the lattice-based structure for buildings can improve sustainability thanks to more efficient factory-based production process, compared to onsite labour and material, reducing the whole-life cost of built environment. The offsite production of lattices for buildings home can not only decrease the embodied energy; more precise and flexible factory production environment enables more creativity and deliver highquality homes faster, more reliably and at the same cost as masonrybuilt one. Furthermore, the thermal efficiency of the building can be much higher than that of made from conventional building blocks making them more environmentally friendly.

\section{Declaration of competing interest}

The authors declare that they have no known competing financial interests or personal relationships that could have appeared to influence the work reported in this paper.

\section{Acknowledgement}

The authors acknowledge the HEIF funding offered by the De Montfort University for this research.

\section{References}

[1] M. Bouquerel, T. Duforestel, D. Baillis, G. Rusaouen, Heat transfer modeling in vacuum insulation panels containing nanoporous silicas - a review, Energy Build. 54 (2012) 320-336, https://doi.org/10.1016/j.enbuild.2012.07.034.

[2] L. Gong, Y. Wang, X. Cheng, R. Zhang, H. Zhang, Thermal conductivity of highly porous mullite materials, Int. J. Heat Mass Tran. 67 (2013) 253-259, https://doi. org/10.1016/j.ijheatmasstransfer.2013.08.008.

[3] Y. Zhang, K. Du, J. He, L. Yang, Y. Li, S. Li, Impact factors analysis on the thermal performance of hollow block wall, Energy Build. 75 (2014) 330-341, https://doi. org/10.1016/j.enbuild.2014.02.037.

[4] A. Mirabolghasemi, A.H. Akbarzadeh, D. Rodrigue, D. Therriault, Thermal conductivity of architected cellular metamaterials, Acta Mater. 174 (2019) 61-80, https://doi.org/10.1016/j.actamat.2019.04.061.

[5] M.V. Shoubi, M.V. Shoubi, A. Bagchi, A.S. Barough, Reducing the operational energy demand in buildings using building information modeling tools and sustainability approaches, Ain Shams Eng. J. 6 (2015) 41-55, https://doi.org/ 10.1016/j.asej.2014.09.006.

[6] M.K. Dixit, Life cycle recurrent embodied energy calculation of buildings: a review, J. Clean. Prod. 209 (2019) 731-754, https://doi.org/10.1016/j jclepro.2018.10.230.

[7] A. Stephan, L. Stephan, Life cycle energy and cost analysis of embodied, operational and user-transport energy reduction measures for residential buildings, Appl. Energy 161 (2016) 445-464, https://doi.org/10.1016/j. apenergy.2015.10.023.

[8] A.A. Morini, M.J. Ribeiro, D. Hotza, Early-stage materials selection based on embodied energy and carbon footprint, Mater. Des. 178 (2019) 107861, https:// doi.org/10.1016/j.matdes.2019.107861.

[9] A. Takezawa, X. Zhang, M. Kitamura, Optimization of an additively manufactured functionally graded lattice structure with liquid cooling considering structural performances, Int. J. Heat Mass Tran. 143 (2019), https://doi.org/10.1016/j. cemconres.2018.06.001.

[10] R.R.J. Sélo, S. Catchpole-Smith, I. Maskery, I. Ashcroft, C. Tuck, On the thermal conductivity of AlSi10Mg and lattice structures made by laser powder bed fusion, Addit. Manuf. 34 (2020), https://doi.org/10.1016/j.compstruct.2019.01.103.

[11] W. Liu, H. Song, C. Huang, Experimental and numerical modelling of mechanical properties of 3D printed honeycomb structures, Addit. Manuf. 35 (2020), https:// doi.org/10.1016/j.measurement.2017.11.037. 
[12] J. Plocher, A. Panesar, Review on design and structural optimisation in additive manufacturing: towards next-generation lightweight structures, Mater. Des. 183 (2019) 108164, https://doi.org/10.1016/j.matdes.2019.108164.

[13] A. Radman, X. Huang, Y.M. Xie, Topological design of microstructures of multiphase materials for maximum stiffness or thermal conductivity, Comput. Mater. Sci. 91 (2014) 266-273, https://doi.org/10.1016/j.commatsci.2014.04.064.

[14] J.Y. Ho, K.C. Leong, T.N. Wong, Additively-manufactured metallic porous lattice heat exchangers for air-side heat transfer enhancement, Int. J. Heat Mass Tran. 150 (2020), https://doi.org/10.1016/j.compositesb.2014.03.015.

[15] K. Wei, R. He, X. Cheng, Y. Pei, R. Zhang, D. Fang, Fabrication and heat transfer characteristics of $\mathrm{C} / \mathrm{SiC}$ pyramidal core lattice sandwich panel, Appl. Therm. Eng. 81 (2015) 10-17, https://doi.org/10.1016/j.applthermaleng.2015.02.012.

[16] H. Wadley, Cellular metals manufacturing, Adv. Eng. Mater. 4 (2002) 726-733.

[17] T. Kim, C.Y. Zhao, T.J. Lu, H.P. Hodson, Convective heat dissipation with latticeframe materials, Mech. Mater. 38 (2004) 767-780.

[18] C.C. Seepersad, J.K. Allen, D.L. McDowell, F. Mistree, Multifunctional topology design of cellular material structures, J. Mech. Des. 130 (2008).

[19] J. Song, W. Zhou, Y. Wang, R. Fan, Y. Wang, J. Chen, Y. Lu, Octet-truss cellular materials for improved mechanical properties and specific energy absorption, Mater. Des. 173 (2019).

[20] S. Pérez-Tamarit, A.H.E. Solórzano, I. Manke, M. Rodríguez-Péreza, Effect of solid phase corrugation on the thermo-mechanical properties of low density flexible cellular polymers, Mater. Des. 161 (2019).

[21] A. Bhattacharya, V.V. Calmidi, R.L. Mahajan, Thermophysical properties of high porosity metal foams, Int. J. Heat Mass Tran. 45 (2002) 1017-1031, https://doi. org/10.1016/S0017-9310(01)00220-4.

[22] J. Fricke, X. Lu, P. Wang, D. Büttner, U. Heinemann, Optimization of monolithic silica aerogel insulants, Int. J. Heat Mass Tran. 35 (1992) 2305-2309, https://doi org/10.1016/0017-9310(92)90073-2.

[23] A.G. Leach, The thermal conductivity of foams. I. Models for heat conduction, J. Phys. D Appl. Phys. 26 (1993) 733-739, https://doi.org/10.1088/0022-3727/ 26/5/003.

[24] M.F. Ashby, The properties of foams and lattices, Philos. T. R. Soc. A. 364 (2006) 15-30, https://doi.org/10.1098/rsta.2005.1678.

[25] E. Cuce, P.M. Cuce, Vacuum glazing for highly insulating windows: recent developments and future prospects, Renew. Sustain. Energy Rev. 54 (2016) 1345-1357, https://doi.org/10.1016/j.rser.2015.10.134.

[26] T.W. Clyne, I.O. Golosnoy, J.C. Tan, A.E. Markaki, Porous materials for thermal management under extreme conditions, Philos. Trans. Royal Soc. A. 364 (2006) 125-146, https://doi.org/10.1098/rsta.2005.1682.

[27] K. Bakker, H. Kwast, E.H.P. Cordfunke, The contribution of thermal radiation to the thermal conductivity of porous U02, J. Nucl. Mater. 223 (1995) 135-142, https:// doi.org/10.1016/0022-3115(94)00680-6.

[28] T.J. Lu, L. Valdevit, A.G. Evans, Active cooling by metallic sandwich structures with periodic cores, Prog. Mater. Sci. 50 (2005) 789-815, https://doi.org/ 10.1016/j.pmatsci.2005.03.001.

[29] G.M. Gladysz, K.K. Chawla, Chapter 6 - cellular materials, in: G.M. Gladysz, K. K. Chawla (Eds.), Voids in Materials, Elsevier, Amsterdam, 2015, pp. 103-130, https://doi.org/10.1016/B978-0-444-56367-5.00006-3.

[30] H.C. Tankasala, V.S. Deshpande, N.A. Fleck, Koiter medal paper: crack-tip fields and toughness of two-dimensional elastoplastic lattices, J. Appl. Mech. 82 (2013), https://doi.org/10.1115/1.4030666, 2015.
[31] L. Trhlíková, O. Zmeskal, P. Psencik, P. Florian, Study of the thermal properties of filaments for 3D printing, AIP Conf. Proc. 1752 (2016), 040027, https://doi.org/ 10.1063/1.4955258, 2016.

[32] T. Tábi, I. Sajó, F. Szabo, A. Luyt, J. Kovacs, Crystalline structure of annealed polylactic acid and its relation to processing, Express Polym. Lett. 4 (2010) 659-668, https://doi.org/10.3144/expresspolymlett.2010.80.

\section{Nomenclature \\ Acronyms}

AM: Additive Manufacturing

$C A D$ : Computer-Aided Design

EPS: Expanded Polystyrene

ETC: Effective Thermal Conductivity

Roman Symbols

A: Lattice topology coefficient

$A_{s}$ : Area of the specimen $\left(\mathrm{m}^{2}\right)$

$B$ : Constant for scaling law

C: Constant for rule of mixture

$c_{a}$ : Shape factor

$D_{h}$ : Hydraulic diameter $(\mathrm{mm})$

d: Diameter $(\mathrm{mm})$

Gr: Grashof number

$g$ : Acceleration due to gravity $\left(\mathrm{m} \mathrm{s}^{-2}\right)$

H: Depth $(\mathrm{mm})$

$h$ : Thickness of top/bottom plate of the lattice specimen $(\mathrm{mm})$

$\ell$ : Length of the strut $(\mathrm{mm})$

$m_{s}$ : Mass of the lattice (gram)

$q$ : Heat flux per unit area $\left(\mathrm{Wm}^{-2}\right)$

$T$ : Temperature $(K)$

$T_{\text {avg }}$ : Average air temperature $(K)$

$T_{h, s}$ : Warm side specimen surface temperature $(K)$

$T_{c, s}$ : Cold side specimen surface temperature $(K)$

$\Delta T$ : Temperature difference across the specimen $(K)$

$t$ : Thickness of the strut $(\mathrm{mm})$

$U$ : Thermal transmittance or U-value $\left(\mathrm{Wm}^{-2} \mathrm{~K}^{-1}\right)$

$U_{l}$ : Thermal transmittance or U-value of lattice $\left(W^{-2} \mathrm{~K}^{-1}\right)$

$U_{s}$ : Thermal transmittance or U-value of solid $\left(W^{-2} K^{-1}\right)$

$v_{p}$ : speed of sound in porous media $\left(\mathrm{ms}^{-1}\right)$

$v_{s}$ : speed of sound in solid media $\left(m s^{-1}\right)$

$X, Y, Z$ : Cartesian coordinates

Greek Symbols

$\eta$ : Dynamic viscosity of the air $\left(\mathrm{kg} \mathrm{m}^{-1} \mathrm{~s}^{-1}\right)$

$\lambda_{a}$ : Thermal conductivity of air $\left(W m^{-1} K^{-1}\right)$

$\lambda_{e}$ : Effective thermal conductivity of lattice $\left(W^{-1} K^{-1}\right)$

$\lambda_{s}$ : Thermal conductivity of solid $\left(\mathrm{Wm}^{-1} \mathrm{~K}^{-1}\right)$

$\eta$ : Dynamic viscosity of the air $\left(\mathrm{kg} \mathrm{m}^{-1} \mathrm{~s}^{-1}\right)$

$\rho$ : Density of air $\left(\mathrm{kg} \mathrm{m}^{-3}\right)$

$\bar{\rho}$ : Relative density

$\sigma$ : Boltzmann's constant $\left(W / m^{2} K^{4}\right.$ 